\title{
Takayasu Arteritis - A Case Study
}

\author{
Minal Dhairya Bhavsar ${ }^{1}$, Vinal Charpot ${ }^{2}$ \\ ${ }^{1,2}$ In Charge Lecturer in Government Physiotherapy College, Civil Hospital, Ahmedabad.
}

Corresponding Author: Minal Dhairya Bhavsar

\begin{abstract}
Takayasu arteritis (TA) is a chronic inflammatory disease of unknown etiology. The mechanism of this disease is not exactly defined. The inflammatory process is generally (but not exclusively) initiated in the second or third decade of life through the actions of non-specific inflammatory cells. As the disease progresses, fibrotic stenosis occurs in aorta and its main branches. The consequence of this inflammatory process can be stenosis, thrombosis, dilatation or aneurysm formation in aorta and/ or its branches. In Asia, its incidence (2.69 in a million per year) has been reported to be 100 times higher than in Europe and North America. Because of the delay in diagnosing the disease, patients often experience claudication, absence of pulses, hypertension, myocardial infarction (MI), and cerebrovascular accidents (CVAs). Accurate and early diagnosis of TA can reduce the economic, social, and psychological burdens. We present clinical, laboratory and imaging findings of a 15 years old girl with TA, who initially presented with clinical manifestations of stroke in form of weakness in Lt Side upper and lower limb and aphasia. The rarity of the disease and especially such a presentation can cause considerable delay in the diagnosis and treatment.
\end{abstract}

Keywords: Takayasu arteritis, Cerebrovascular accident, Infarction, physical therapy

\section{INTRODUCTION}

Takayasu arteritis is an idiopathic, chronic, granulomatous inflammation of medium and large arteries with a predilection for aortic arch and its branches. It is for this reason that it is also known as aortic arch syndrome. The progressive inflammation leads to stenosis, fibrosis, thrombosis and aneurysm formation in the involved arteries. It presents usually in females of 2 nd or 3rd decade of life. ${ }^{1,2}$ The various signs and symptoms such as fever, malaise, anorexia, weight loss, extremity pain, claudication, light-headedness, bruits, absent or diminished pulses, hypertension, and loss of blood pressure can be present according to the vessel involvement. ${ }^{3}$

In 1905 Takayasu, professor of ophthalmology at Kanazawa University Japan, presented the case of a 21 year old woman with characteristic fundal arteriovenous anastamoses. ${ }^{4}$ In the same year, Onishi and Kagosha each described similar cases associated with absent radial pulses. ${ }^{5}$ In 1920 , the first postmortem case of a 25 year old woman demonstrated pan arteritis and suggested that the fundal appearances resulted from retinal ischaemia. ${ }^{1}$

Incidence of Takayasu arteritis is rare, but most commonly seen in Japan, South East Asia, India, and Mexico. In 1990, it was included in the list of intractable diseases maintained by the Japanese government, and to date 5000 patients have been registered. A study of North American patients by Hall et al found the incidence to be $2.6 /$ million/ year. ${ }^{6}$ The UK incidence is unknown. Approximately $10-20 \%$ of patients with TA will have ischemic stroke or transient ischemic attacks. ${ }^{7}$ However, the incidence of specific organ involvement differs in the various 
reported series, which may relate to differences in ethnic background. ${ }^{8}$

MRI, magnetic resonance angiography (MRA), Doppler ultrasound, CT, and positron emission tomography (PET) are all potentially useful for evaluation of Takayasu arteritis. Management of Takayasu arteritis is changing. Recent advances in diagnostic clinical tests, especially imaging modalities and new biomarkers including reports from our laboratory ${ }^{9}{ }^{10}$, have improved the accuracy of diagnosis, and new immunosuppressive agents are increasingly applied to this disease. Although guidelines for the management of TA were published recently ${ }^{11}$, the clinical utility of these new technologies has not been settled yet.

\section{CASE REPORT}

Here reported case is of 15 years old female suffering from Takayasu arteritis and $\mathrm{CV}$ stroke. She was relatively normal up to the age of 14 years of her age without any significant family history. She had complain of fever since last 4 months and sudden onset of weakness of left side of Upper and lower limb. As the time progressed, she started feeling difficulty in speaking. She had consulted neurophysician for the same and advised for CT scan and MRI. She was unconscious for 2 days and diagnosed having Takayasu arteritis and CV stroke.

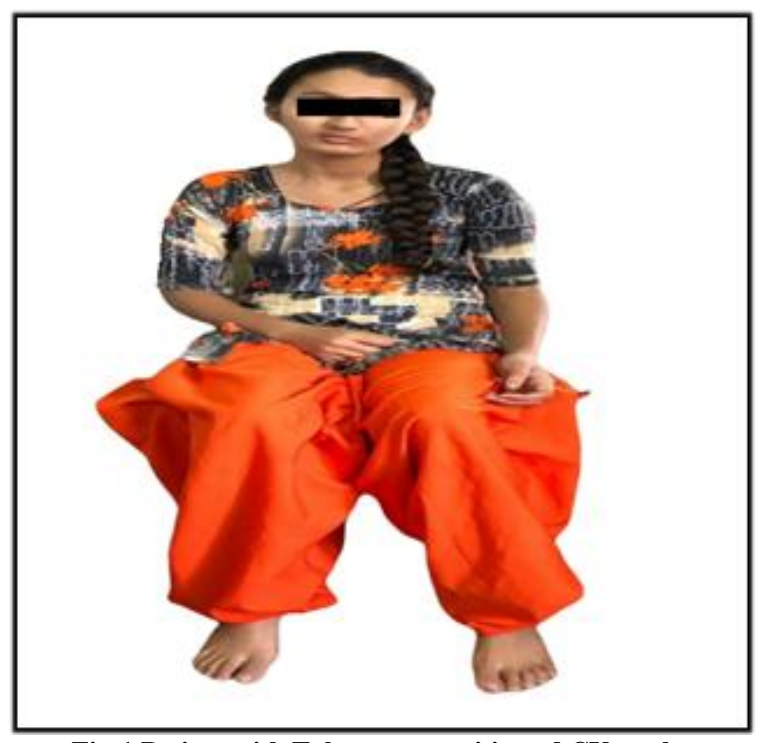

Fig-1 Patient with Takayasu arteritis and CV stroke
Manual muscle testing of Left Upper limb was Zero and Left side lower limb was hip and knee flexors were grade 2 and abductors and adductors were grade 2 . Other muscles have no power. Power of facial muscles was weak functional. Tone was increased in Lt side Upper and lower limb and reflex assessment showed exaggerated Biceps, Triceps, knee and Ankle jerk on Left side. Babinski's sign was positive. Tightness was present in left side calf and hamstring muscles. The left pulse was 90 beats per minute, regular, normal volume, no special character, and no radiofemoral delay. Right-sided carotid artery pulsations were feeble with absent brachial and radial pulses. Right lower limb arterial pulsations were palpable and equal to that of left lower limb. Right carotid bruit was clearly heard on auscultation. The oral temperature was $98^{\circ} \mathrm{F}$ and respiratory rate was 16 breaths per minute and thoracoabdominal. There was no pallor, icterus, cyanosis, pedal edema, clubbing, lymphadenopathy, neck swelling or skin rash.

On CNS examination, the higher mental function was within the normal limit. There was no neck rigidity and no sign of meningeal irritation. Bilateral pupils were normal size. The cranial nerves were not affected. In sensory examination superficial and cortical sensations were intact but deep sensation was affected in Left side upper limb. The muscle bulk was normal and symmetrical bilaterally. Cognitive and perceptual assessment was normal. Chest expansion was reduced.

\section{Investigations}

Her hemoglobin was $9.30 \mathrm{~g} / \mathrm{dL}$, total leukocyte count $13600 / \mathrm{mm} 3$, platelet count $3.68 \mathrm{~L} / \mathrm{mm} 3$, random blood sugar 112 $\mathrm{mg} / \mathrm{dL}$, blood urea $10.80 \mathrm{mg} / \mathrm{dL}$, serum creatinine $0.82 \mathrm{mg} / \mathrm{dL}$, total bilirubin 1.40 $\mathrm{mg} / \mathrm{dL}$, serum sodium $143 \mathrm{meq} / \mathrm{L}$, Vitamin B12 85. Her erythrocyte sedimentation rate (ESR) was persistently high; it was $95 \mathrm{~mm}$. Human immunodeficiency virus (HIV), HBsAg, and anti HCV were non-reactive. 
Anti-nuclear antibody and VDRL were also negative. The chest X-ray and electrocardiogram was within the normal limit.

MR Angiography Report showed complete block in right internal carotid artery, right middle cerebral artery and right posterior communicating artery. Hypo plastic right vertebral artery was noted. Large acute infarct noted in right frontoparieto-temporal region. Mass effect in form of compression over frontal horn of right lateral ventricle and midline shift of $4 \mathrm{~mm}$ towards the left side.
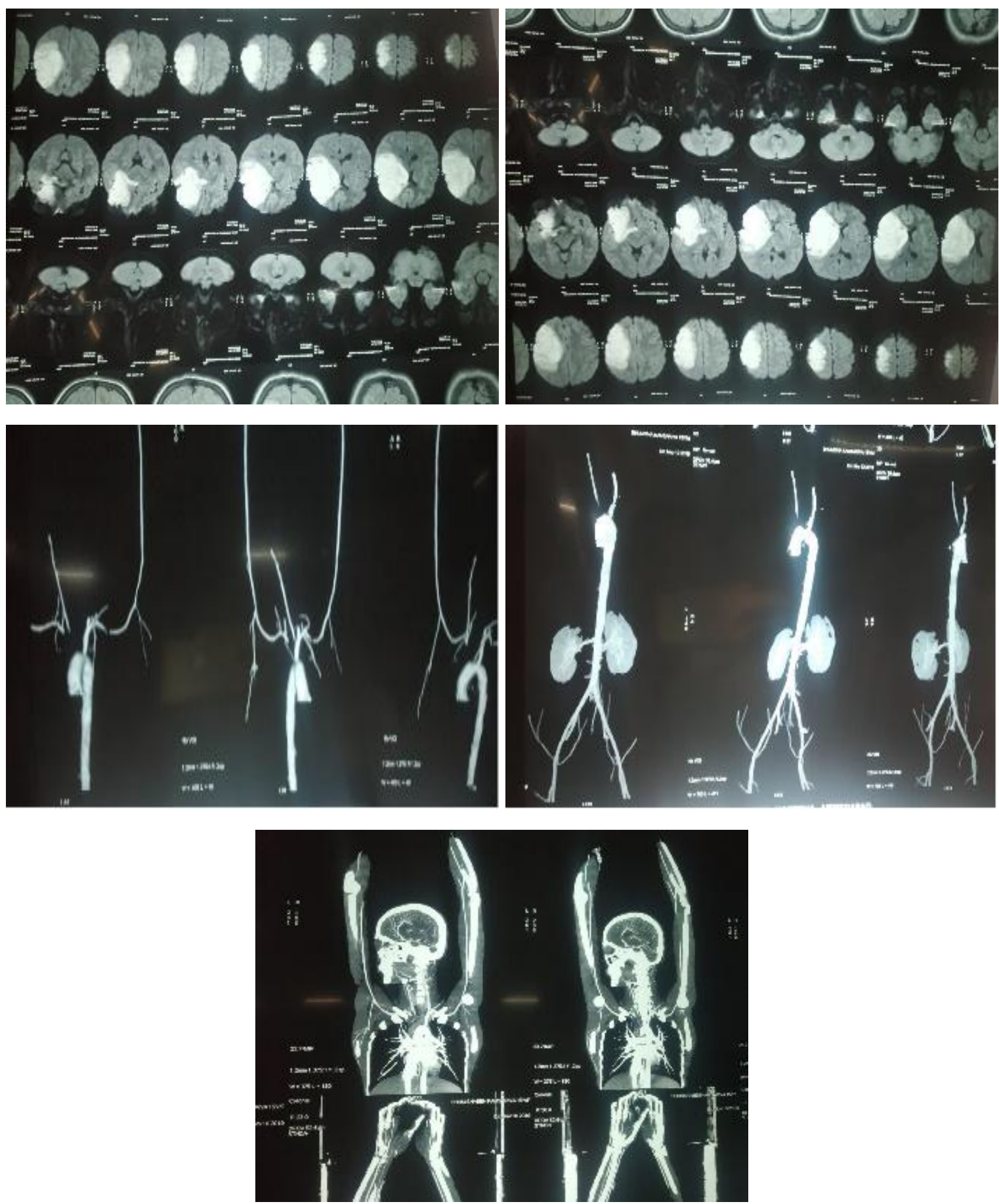

Fig-2 MR angiography of different views

Role of physiotherapy in management of Takayasu arteritis was important to prevent secondary complications. Various studies have shown that both aerobic exercise and resistance exercise training programs have been 
demonstrated to diminish the TNF- $\alpha$ and CRP levels in TA patients. ${ }^{12,13}$

\section{DISCUSSION}

Takayasu arteritis, also known as pulseless disease, thromboaortopathy and Martorell syndrome, is characterized by chronic large-vessel inflammation leading to wall-thickening fibrosis, stenosis and thrombosis, predominantly involving the aorta and its branches. Neurological involvement is not common in Takayasu arteritis and stroke as first presentation of disease is relatively rare. ${ }^{14}$ There is wide variation in the incidence, gender predisposition, disease morbidity and mortality reported in Takayasu arteritis across the globe. Etiology of Takayasu Arteritis is unknown, but recent evidence suggests an association with certain human leukocyte antigen (HLA) alleles and other autoimmune processes like sarcoidosis and inflammatory bowel disease. It is also shown that tuberculosis may have deep association, given a high prevalence of active and past infection in patient with Takayasu arteritis. ${ }^{15}$

Panja's series of 650 cases of Takayasu arteritis, the largest series, reported an incidence of stroke of $22 \%$. In 1990, the American College of Rheumatology proposed the criteria for Takayasu arteritis diagnosis: age at disease onset $\leq 40$ years, claudication of the extremities, decrease brachial artery pulse, systolic blood pressure difference $>10$ $\mathrm{mmHg}$ in upper limbs, bruit over subclavian artery or aorta and radiographic abnormalities. The presence of three out of six criteria is required for a diagnosis and demonstrates a sensitivity of $90.5 \%$ and specificity of $97.8 \% .^{16}$

Doppler ultrasound, CT angiography (CTA), and recently magnetic resonance angiography (MRA) have shown promise in the diagnosis of Takayasu arteritis. ${ }^{17}$

MRA provides high resolution details of vessel wall thickness and lumen configuration. It allows the measurement of wall enhancement as a reflection of edema and inflammation. Compared to the gold standard of angiography, $2 \%$ of stenosed vessels are over estimated as occluded on MRA. By reduction of enhancement on follow up, MRA also serves as a surrogate marker for disease activity. Steroids are the mainstay of treatment and approximately half of the patients respond to it. Steroidsunresponsive patients can be treated with cytotoxic drugs like cyclophosphamide, azathioprine and methotrexate. Some studies have reported the use of several immunosuppressive agents such as methotrexate, Mycophenolate mofetil and azathioprine. ${ }^{18}$ Anti-TNF therapy has also been used successfully in a few patients. ${ }^{19}$ Surgical treatment is offered to those with severe stenosis of renal artery, extremity claudication, stenosis of three or more cerebral vessels or evidence of coronary artery involvement.

Here our patient was diagnosed with Takayasu arteritis and Left side hemiparesis along with facial muscle involvement. So role of physiotherapy was to maintain available strength of muscle and to prevent secondary complications like contracture, tightness and deformity in left side upper and lower limb. For improvement of range of motion passive range of motion exercise, active assisted range of motion exercise and strengthening exercise was given to the patient. For sensory reeducation Rood's approach and weight bearing exercise in all position was given. Along with this balanced training in sitting and standing position, postural correction and gait training by mirror biofeedback was given to the patient. For facial muscle reeducation electrical stimulation, strengthening exercise and kinesio taping was given to the patient.

Early intervention in stroke patients by physical rehabilitation like physiotherapy helps in faster recovery. Thus, stroke patients with the potential for functional recovery could receive regular follow-up physiotherapy treatment, which could help them to reduce economic burden and can prevent negative effects and immobilityrelated complications. ${ }^{20}$ 


\section{Acknowledgement: None}

Conflict of Interest: There was no conflict of interest at personal or institutional level

\section{Source of Funding: Not required}

Ethical Clearance:-Informed written signed consent was taken from the patient.

\section{REFERENCES}

1. Numano $\mathbf{F}$, Okawara $\mathrm{M}$, Inomata $\mathrm{H}$ et al. Takayasu's arteritis. Lancet 2000; 356: 1023-25.

2. Koide K. Takayasu arteritis in Japan. Heart Vessels Suppl 1992; 7: 48-54.

3. Kerr GS.Takayasu's arteritis.Rheum DisClin NorthAm1995; 21:1041-58.

4. Takayasu M. A case with peculiar changes of the retinal central vessels [in Japanese]. Acta of the Ophthalmic Society of Japan 1908; 12:554-5.

5. Numano F, Kakuta T. Takayasu arteritisfive doctors in the history of Takayasu arteritis. Hall S, Barr W, Lie JT, et al.

6. Takayasu arteritis A study of 32 North American patients. Medicine. 1985; 64: 8999.Int J Cardiol 1996;54:S1-10

7. Kerr GS, Hallahan CW, Giordano J et al. Takayasu arteritis. Ann Intern Med 1994; 120:919-29.

8. Moriwaki R, Noda M, Yajima M, Sharma BK, Numano F. Clinical manifestations of takayasu arteritis in India and Japan - new classification of angiographic findings. Angiology 1997; 48:369-79.

9. Tezuka D, Haraguchi G, Ishihara T, et al. Role of FDG-PET/CT and utility of maximum standard uptake value in Takayasu arteritis: sensitive detection of recurrence. J Am Coll Cardiol Imaging 2012; 5:422-9.

10. Ishihara $T$, Haraguchi $G$, Kamiishi $T$, Tezuka D, Inagaki $H$, Isobe $M$. Sensitive assessment of activity of Takayasu's arteritis by pentraxin3, a new biomarker. J Am Coll Cardiol 2011; 57:1712-3.

11. Japanese Circulation Society Joint working Group. Guideline for management of vasculitis syndrome (Japanese Circulation Society 2008). Circ J 2011; 75:474-503.
12. Oliveira DS, Shinjo SK, Silva MG, de SaPinto AL, Lima FR, Roschel $\mathrm{H}$, et al. . Exercise in Takayasu arteritis: effects on inflammatory and antigenic factors and disease-related symptoms. Arthritis Care Res (Hoboken). (2017) 69:892-902. 10.1002/acr.23011

13. Li G, Liu F, Wang Y, Zhao M, Song Y, Zhang L. Effects of resistance exercise on treatment outcome and laboratory parameters of Takayasu arteritis with magnetic resonance imaging diagnosis: a randomized parallel controlled clinical trial. Clin Cardiol. (2020) 43:1273-8. 10.1002/clc.23439

14. Sikaroodi H, Motamedi M, Kahnooji H et al. Stroke as the first manifestation of Takayasu arteritis. Acta Neurol Belg 2007 Mar; 107(1): 18-21.

15. Johnston SL, Lock RJ, Gompels MM. Takayasu arteritis: A review. J Clin Pathol 2002; 55: 481-86.

16. Arend WP, Michel BA, Bloch DA et al. The American college of Rheumatology 1990 criteria for the classification of Takayasu arteritis. Arthritis Rheum 1990 Aug; 33(8): 1129-34

17. Choe YH, Kim DK, Koh EM et al. Takayasu'sarteritis: Diagnosis with MR imaging and MR angiography in acute and chronic active stages. J Magn Reson Imaging 1999; 10: 751-57.

18. Valsakumar AK, Valappil UC, Jorapur V et al. Role of immunosuppressive therapy on clinical, immunological, and angiographic outcome in active Takayasu's arteritis. J Rheumatol 2003 Aug; 30(8): 1793-98.

19. Hoffman GS, Merkel PA, Brasington RD et al. Antitumor necrosis factor therapy in patients with difficult to treat Takayasu arteritis. Arthritis Rheum 2004 Jul; 50(7): 2296-2304.

20. Gargi Dey, Jyothi $\mathrm{Rm}$ et al. retrospective observational study of the effect of physiotherapy treatment on outcomes in stroke patients. National journal of physiology, pharmacy and pharmacology 2019; vol 9 (8).

How to cite this article: Bhavsar MD, Charpot V. Takayasu arteritis- a case study. Int $J$ Health Sci Res. 2021; 11(6): 362-366. DOI: https://doi. org/10.52403/ijhsr.20210654 